\title{
Protein kinase A suppresses the differentiation of 3T3-L1 preadipocytes
}

\author{
Fuqiang $\mathrm{Li}^{1}$, Dongmei Wang ${ }^{1}$, Yiran Zhou ${ }^{1}$, Bo Zhou ${ }^{1}$, Yanan Yang ${ }^{1}$, Hehua Chen ${ }^{1}$, Jianguo Song ${ }^{1}$
}

${ }^{1}$ Laboratory of Molecular Cell Biology, Institute of Biochemistry and Cell Biology, Shanghai Institutes for Biological Sciences, Chinese Academy of Sciences, 320 Yueyang Road, Shanghai 200031, China

cAMP and protein kinase A (PKA) are widely known as signaling molecules that are important for the induction of adipogenesis. Here we show that a strong increase in the amount of cAMP inhibits the adipogenesis of 3T3-L1 fibroblast cells. Stimulation of PKA activity suppresses adipogenesis and, in contrast, inhibition of PKA activity markedly accelerates the adipogenic process. As adipogenesis progresses, there is a significant increase in the expression level of PKA regulatory subunits and a corresponding decrease in PKA activity. Moreover, treatment of 3T3-L1 cells with epidermal growth factor (EGF) stimulates PKA activity and blocks adipogenesis. Inhibition of PKA activity abolishes this suppressive effect of EGF on adipogenesis. Moreover, activation of PKA induces serine/threonine phosphorylation, reduces tyrosine phosphorylation of insulin receptor substrate 1 (IRS-1) and the association between PKA and IRS-1. Taken together, our study demonstrates that PKA has a pivotal role in the suppression of adipogenesis. cAMP at high concentrations can suppress adipogenesis through PKA activation. These findings could be important and useful for understanding the mechanisms of adipogenesis and the relevant physiological events.

Keywords: cAMP, PKA, adipocyte, adipogenesis, C/EBP, PPAR

Cell Research (2008) 18:311-323. doi: 10.1038/cr.2008.12; published online 15 January 2008

\section{Introduction}

White adipose tissue is not only important for energy storage but also an endocrine organ that regulates energy homeostasis [1]. Obesity and diabetes have been associated with adipocytes [2]. In white adipose tissue, preadipocytes exist in close proximity to adipocytes; preadipocytes can modify the function of adipose tissue by differentiating into adipocytes. The study of adipogenesis has been greatly facilitated by establishing immortal preadipocyte cell lines, such as 3T3-L1 preadipocytes. The adipocytes differentiated from these cells exhibit most of the key features of adipocytes in vivo [3]. In general, the induction of adipogenesis

Correspondence: Jianguo Song

Tel/Fax: +86-21-54921167

E-mail: jgsong@sibs.ac.cn

Abbreviations: peroxisome proliferator-activated receptor (PPAR); CCAAT/ enhancer-binding protein (C/EBP); methylisobutylxanthine, dexamethasone and insulin (MDI); protein kinase A (PKA); insulin receptor substrate (IRS)

Received 19 July 2007; accepted 12 September 2007; published online 15 January 2008 is initiated by treating confluent 3T3-L1 preadipocytes with methylisobutylxanthine, dexamethasone and insulin (MDI). The post-confluence mitotic clonal expansion of cells from $\mathrm{G}_{0} / \mathrm{G}_{1}$ growth arrest is first induced by MDI, and after that the cells exit the cell cycle and commit to terminal differentiation. During adipogenesis, two transcription factors, CCAAT/enhancer-binding protein- $\beta(\mathrm{C} / \mathrm{EBP} \beta)$ and $\mathrm{C} / \mathrm{EBP} \delta$, are rapidly induced, leading to the induction of expression of the key adipogenic transcription factors $\mathrm{C} / \mathrm{EBP} \alpha$ and peroxisome proliferator-activated receptor- $\gamma$ (PPAR $\gamma$ ), and the subsequent induction of expression of the specific genes that are necessary to bring about the adipocyte phenotype [4-6].

Knowledge about the biological function of cAMP has been increasing tremendously since the discovery of cAMP in the 1960s. cAMP functions mainly through activating cAMP-dependent protein kinase A(PKA), Epacs (exchange proteins directly activated by cAMP) $[7,8]$ and cyclic nucleotide-gated ion channels $[9,10]$. A large number of PKA physiological substrates have been identified [11]. These substrates can be detected in various parts of cells. PKA is the main target of cAMP, which exists either as an inactive holoenzyme composed of two regulatory $(\mathrm{R})$ 
and two catalytic $(\mathrm{C})$ subunits $\left(\mathrm{R}_{2} \mathrm{C}_{2}\right)$, or as dissociated subunits. The $\mathrm{R}$ subunits inhibit the activity of the $\mathrm{C}$ subunits by binding to the $\mathrm{C}$ subunits, and cAMP stimulates PKA activity by binding to the $\mathrm{R}$ subunits, which causes the dissociation of the $\mathrm{R}$ subunits from the $\mathrm{C}$ subunits. Therefore, the R subunits can be considered as inhibiting proteins for the $\mathrm{C}$ subunits of PKA. In other words, PKA activity can be regulated by the synthesis or by the degradation of the R subunits of PKA. Thus, increased synthesis of the $\mathrm{R}$ subunits of PKA increases the formation of the inactive holoenzyme of PKA, whereas degradation of the $\mathrm{R}$ subunits of PKA can lead to release and activation of the $\mathrm{C}$ subunits of PKA. The effect of cAMP signaling on the differentiation of preadipocytes has been a focus of interest in adipogenic studies in recent years. As one of the inducers for adipogenesis, methylisobutylxanthine (MIX) is a broad-spectrum phosphodiesterase (PDE) inhibitor that can elevate intracellular level of cAMP. For this reason, cAMP has been considered as an inducer for adipogenesis, and PKA, a classical target of cAMP, was also regarded as a molecule that stimulates the differentiation of preadipocytes. However, no direct study has been carried out to determine the exact role of PKA on adipogenesis.

Insulin has been widely reported to promote adipogenesis [12]. Divergent insulin signaling pathways can be transmitted by insulin receptor substrate (IRS) proteins [13]. The tyrosine phosphorylation of IRS by insulin receptor signaling activates IRS, which is essential for the adipogenic effect of insulin. Furthermore, IRS-1 has been reported to have an important role in adipogenesis. IRS-1 deficiency in embryonic fibroblast cells leads to inhibition of adipogenesis in these cells [14]. There are serine/threonine phosphorylation sites in IRS-1, and the phosphorylation of these sites inhibits IRS-1 [15-17], leading to insulin resistance. IRS-1 is therefore related to obesity and diabetes $[18,19]$.

In this study, we characterized the regulatory effect of cAMP and PKA on the differentiation of preadipocytes, and investigated the potential mechanism through which PKA exerts its effect on adipogenesis.

\section{Results}

\section{The effect of cAMP on adipogenesis}

To investigate the effect of cAMP on adipogenesis, we examined whether the cell-permeable cAMP-elevating agent forskolin can substitute for MIX in stimulating adipogenesis. As shown in Figure 1A and 1B (A: Oil Red O staining of the lipid drops; B: expression levels of PPAR $\gamma$ and $\mathrm{C} / \mathrm{EBP} \alpha$ ), forskolin at high concentrations can significantly increase DI (dexamethasone and insulin)-induced adipogenesis (about 50-70\%). However, low concentrations of cAMP had no or little effect on adipogenesis. Consistently, the cAMP analog 8-Br-cAMP also increased adipogenesis (Figure $1 \mathrm{C}$ and $1 \mathrm{D}$ ). The maximum stimulation by 8 -Br-cAMP $(200 \mu \mathrm{M})$ and DI can be as high as that induced by MDI. These results indicate that cAMP or forskolin can replace or partly replace MIX in stimulating adipogenesis. For understanding the exact role of cAMP in adipogenesis, we also detected the effect of forskolin and MIX on cAMP production in 3T3-L1 preadipocytes. As shown in Figure 1E, forskolin greatly increases the level of intracellular cAMP, which in this case reached about 120 -fold (the control is $1.1 \mathrm{pmol} / \mathrm{ml}$ ) within a few minutes and then dropped to and was maintained at a level of about 20 -fold. Subsequently, we measured the effect of forskolin on the production of intracellular cAMP during adipogenesis. As shown in Figure 1F, cAMP levels increased to about 10 -fold during the first $4 \mathrm{~h}$ of induction by MDI, and a much greater increase in cAMP levels was induced by forskolin under the same conditions. Interestingly, pretreatment of cells with forskolin inhibited adipogenesis in a dose-dependent manner. At a concentration of $1 \mu \mathrm{M}$, adipogenesis was completely suppressed (Figure 1G). The same suppressive effect of forskolin was also observed by determining the expression levels of $\mathrm{C} / \mathrm{EBP} \alpha$ and PPAR $\gamma$

Figure 1 The effect of 8-Br-cAMP and forskolin on adipogenesis. 3T3-L1 preadipocytes were exposed to agents as indicated in the absence of MIX $(0.5 \mathrm{mM})$ together with $\mathrm{DI}$ (dexamethasone and insulin) (at day 0 ) for 2 days. They were then incubated in fresh medium supplemented with insulin $(1 \mu \mathrm{g} / \mathrm{ml})$ for another 2 days. They were then fed with fresh medium every second day. On days 6 and 8, adipogenesis was determined by Oil Red $\mathrm{O}$ staining and the expression of C/EBP $\alpha$ and PPAR $\gamma$. (A and B) The effect of forskolin on adipogenesis. (C and D) The effect of 8-Br-cAMP on adipogenesis. (E) Forskolin elevates cAMP levels. 3T3-L1 preadipocytes were treated with forskolin (Forsk, $10 \mu \mathrm{M}$ ) for the indicated times, cells were lysed and the cAMP levels were determined as described in the Materials and Methods section. Data in the inset are the means \pm standard deviations (SDs) from three independent experiments. (F) Effect of forskolin on CAMP levels during adipogenesis. 3T3-L1 preadipocytes were induced with MDI in the presence or absence of forskolin $(1 \mu \mathrm{M})$, and the cAMP levels were determined at the indicated time points. Data in the inset are the means \pm SDs from three independent experiments. ${ }^{* * *} P<0.001$ versus cells induced with MDI in the absence of forskolin. (G and $\mathbf{H}$ ) Forskolin inhibits MDIinduced adipogenesis. 3T3-L1 preadipocytes were pretreated with or without forskolin for 15 min, and adipogenesis was induced with MDI. Adipogenesis was determined as described above. (I and $\mathbf{J}$ ) 8-Br-cAMP inhibits MDI-induced adipogenesis. 
A

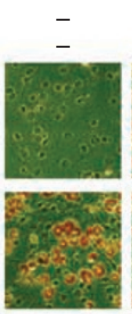

DI

Forskolin $(0.1 \mu \mathrm{M})$
DI

Forskolin

$(1 \mu \mathrm{M})$
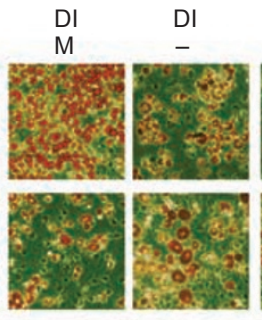

DI

orskolin Forskolin $(10 \mu \mathrm{M}) \quad(20 \mu \mathrm{M})$

C

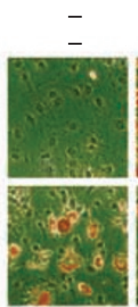

$\mathrm{DI}$

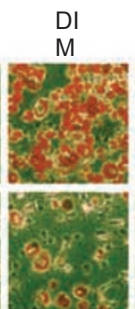

DI

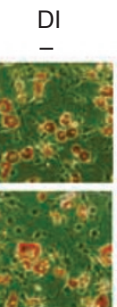

DI

8-Br-CAMP 8-Br-CAMP 8-Br-CAMP 8-Br-cAMP

$\begin{array}{llll}(1 \mu \mathrm{M}) & (10 \mu \mathrm{M}) & (100 \mu \mathrm{M}) & (200 \mu \mathrm{M})\end{array}$

$E$
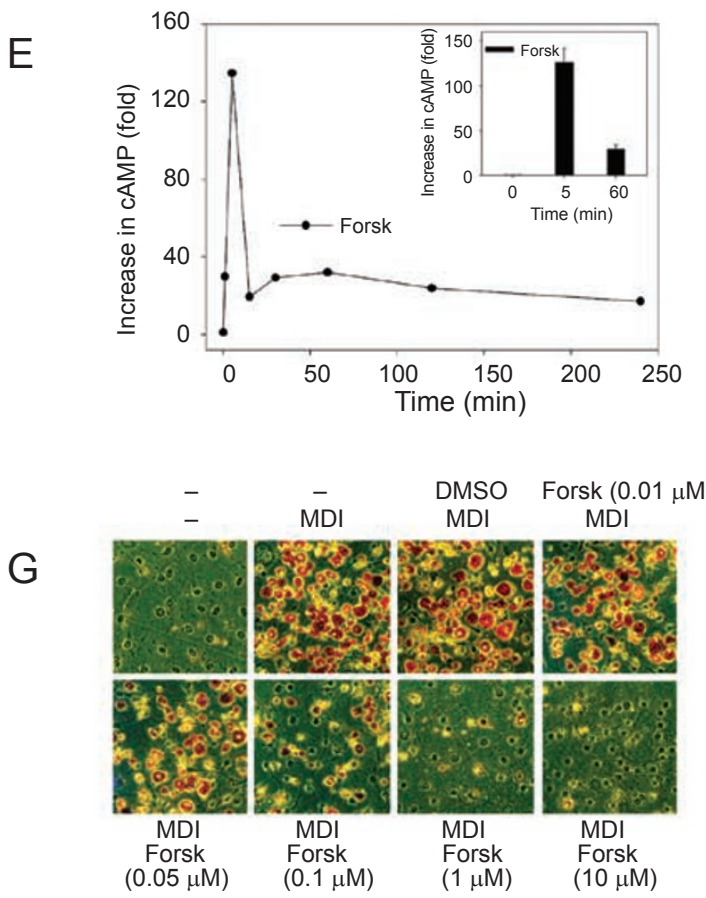

DI
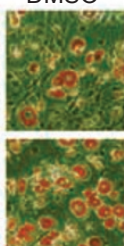

DI
B

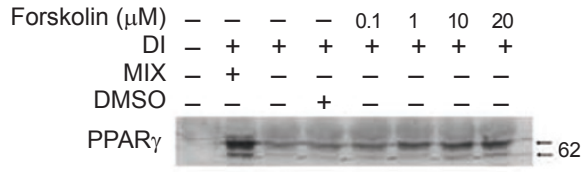

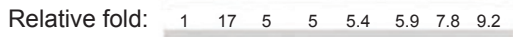

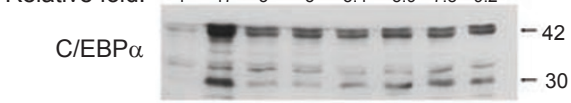

$\begin{array}{lllllllll}\text { Relative fold: } & 1 & 12.7 & 3.2 & 3.5 & 3.3 & 4.6 & 5.2 & 6.1\end{array}$

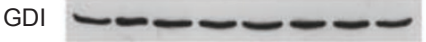

8-Br-cAMP $(\mu \mathrm{M})-\quad+\quad-\quad 110100200$

D

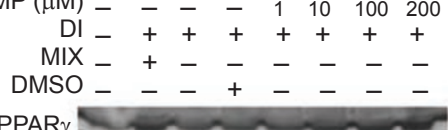

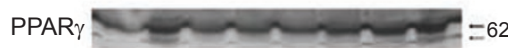

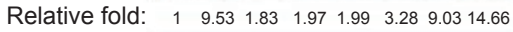

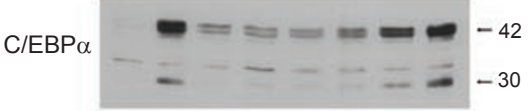

$\begin{array}{llllllll}\text { Relative fold: } \quad 1 & 32.5 & 6.2 & 6.5 & 6.7 & 10.7 & 31.7 & 52.1\end{array}$

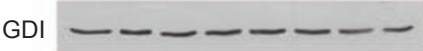

\section{$\mathrm{F}$}

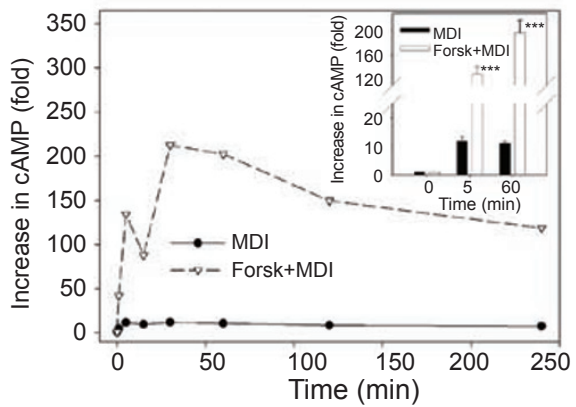

$\mathrm{H} \quad$ Forsk (uM) - $\quad-0.010 .050 .1 \quad 1 \quad 10$

MDI -++++++

DMSO - $-+\ldots+\ldots 47.5$

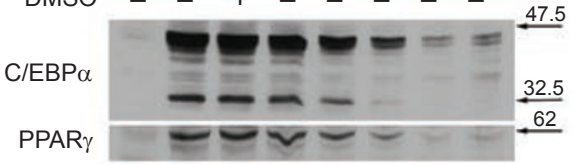

$\mathrm{J}$

8-Br-cAMP $(\mu \mathrm{M})-\quad-\quad \begin{array}{llllll} & 5 & 10 & 50 & 100200\end{array}$

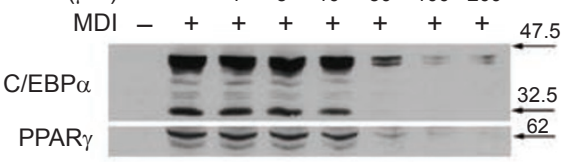


(Figure 1H). Moreover, treatment of cells with 8-bromocAMP also suppressed adipogenesis in a dose-dependent manner as determined by either the morphological phenotype (Figure 1I) or the expression levels of C/EBP $\alpha$ and PPAR $\gamma$ (Figure 1J). These findings suggest that a strong and sustained increase in cAMP levels can inhibit the process of adipogenesis.

The effect of downregulating cAMP on MDI-induced adipogenesis

In addition to the above evidence, we treated 3T3-L1 cells with SQ22536, a specific adenylate cyclase inhibitor that can significantly reduce intracellular cAMP levels (Figure 2A). As shown in Figure 2, pretreatment of cells with SQ22536 produces no inhibitory effect on adipogenesis, as examined by immunoblotting C/EBP $\alpha$ and PPAR $\gamma$ (Figure 2B), or by Oil Red O staining (Figure 2C). Further investigation showed that SQ22536 can actually promote the adipogenesis of 3T3-L1 preadipocytes because it significantly reduces the induction time required for adipogenesis (Figure 2D). The expression levels of C/EBP $\alpha$ and PPAR $\gamma$ in cells pretreated with SQ22536 and exposed to MDI for $36 \mathrm{~h}$ was higher than that in cells without SQ22536 pretreatment but exposed to MDI for the typical $48 \mathrm{~h}$.

\section{Inhibiting PKA promotes MDI-induced adipogenesis}

It has been shown that adipogenesis could be promoted mostly through increasing the amount of triacylglycerol storage or by reducing the induction time required for adipogenesis [20,21]. Since PKA activity can be strongly regulated by cAMP, we also investigated the role of PKA in adipogenesis. We firstly determined the effect of PKA on the induction time of adipogenesis. Rp-cAMP, an agent that inhibits PKA activity, can reduce the required MDI induction time from 48 to $36 \mathrm{~h}$ (Figure 3A). PKA activity can be determined by examining the phosphorylation of the cAMP-response element binding protein (CREB). H89, a widely used PKA inhibitor, significantly reduces the basal level of PKA activity as determined by the phosphorylation of CREB (Figure 3B), indicating an inhibitory effect of H89 on PKA activity. In the presence of H89, MDI-induced adipogenesis was brought forwards by 2 days (Figure 3C), and an increase in the amount of triacylglycerol storage could also be observed. The same effect of H89 on the adipogenesis of 3T3-L1 preadipocytes was also determined by examining the expression levels of $\mathrm{C} / \mathrm{EBP} \alpha$ and PPAR $\gamma$ (Figure 3D). To further confirm the results shown above, protein kinase A inhibitor (PKI), a protein that can specifically inhibit PKA activity both in vitro and in vivo, was transfected into 3T3-L1 preadipocytes (Figure 3E), which effectively inhibited PKA activity (Figure 3F). The results show that PKI transfection also promotes adipogenesis as
A

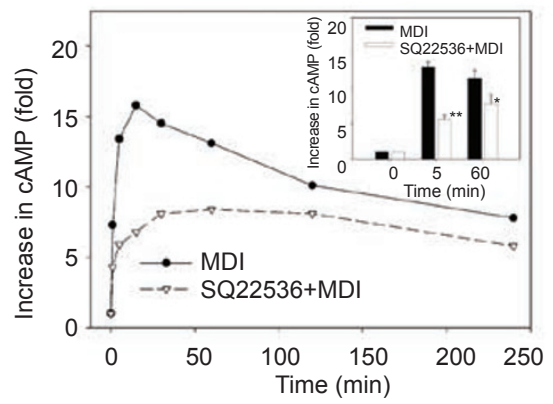

B
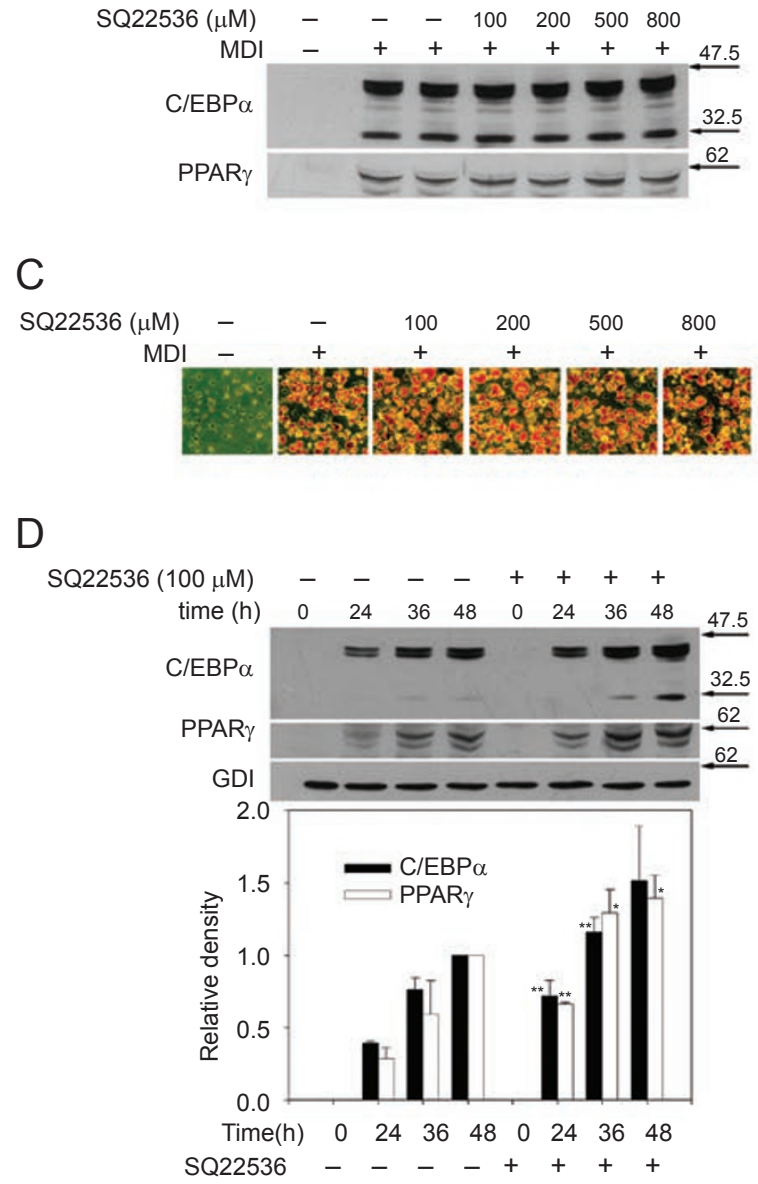

Figure 2 The effect of downregulation of cAMP levels on MDIinduced adipogenesis. Cell treatments and adipogenesis induction are as described in Materials and Methods. (A) Inhibition of MDI-mediated cAMP production by the specific adenylate cyclase inhibitor SQ22536. 3T3-L1 preadipocytes were induced with MDI in the absence or presence of SQ22536 $(500 \mu \mathrm{M})$ for the times indicated. Data in the inset are the means \pm SDs from three independent experiments. ${ }^{*} P<0.05$; ${ }^{* *} P<0.01$ versus controls (not treated with SQ22536). (B and C) Dose effect of SQ22536 on MDI-induced adipogenesis. (D) SQ22536 reduces the induction time required for adipogenesis. Upper panel: immunoblotting of C/EBP $\alpha$ and PPAR $\gamma$. Lower panel: data are the means \pm SDs from three independent experiments. ${ }^{*} P<0.05$; ${ }^{* *} P<0.01$ versus controls (not treated with SQ22536). 
examined by triacylglycerol storage (Figure 3G) and the expression levels of $\mathrm{C} / \mathrm{EBP} \alpha$ and PPAR $\gamma$ (Figure $3 \mathrm{H}$ ). These results indicate that PKA has a suppressive role in the adipogenesis of 3T3-L1 cells.

\section{PKA activity and its expression levels during adipogen- esis}

To confirm the role of PKA in adipogenesis, we also detected the PKA activity at different time points during adipogenesis. PKA activity increased in the first 2 days of induction, but decreased markedly from the third day (Figure 4A). These results suggest that a downregulation of PKA activity is required for adipogenesis to proceed. To investigate the potential relationship between the changes in PKA activity and the expression levels of PKA regulatory subunits, we examined the expression levels of several types of regulatory subunits of PKA during adipogenesis. As shown in Figure 4B, the expression of the RI and RII $\alpha$ subunits of PKA increased significantly, but no increase in RII $\beta$ or the catalytic subunits of PKA was observed. Interestingly, upregulation of the expression of the PKA regulatory subunits began on the third day, which probably accounts for the corresponding decrease in PKA activity. It is apparent that downregulation of PKA activity is an essential signaling event required for cells to initiate adipogenesis. Increased expression of the PKA regulatory subunits may therefore suppress the PKA activity that is important for adipogenesis.

\section{The effect of PKA activation or inhibition on adipogen- esis}

Unlike the 8-bromo-cAMP, which can activate PKA, Rp-cAMP is a cAMP analog that cannot activate PKA, and 8-CPT-2'-O-Me-cAMP is a cAMP analog that can activate Epac but not PKA. To determine whether cAMP suppresses adipogenesis through PKA, we also determined the effect of Rp-cAMP and 8-CPT-2'-O-Me-cAMP on adipogenesis by examining the triacylglycerol storage (Figure $5 \mathrm{~A}$ ) or expression levels (Figure $5 \mathrm{~B}$ ) of $\mathrm{C} / \mathrm{EBP} \alpha$ and PPAR $\gamma$. As shown on Figure 5, only 8-bromo-cAMP suppressed adipogenesis; neither Rp-cAMP nor 8-CPT-2'O-Me-cAMP showed any inhibitory effect on this process, indicating that PKA has a role in cAMP-mediated suppression of adipogenesis. To verify this function of PKA, we transfected the PKA catalytic subunit- $\alpha$ (PKA C $\alpha$ ) onto 3T3-L1 preadipocytes (Figure 5C), and then examined the effect on adipogenesis. The results showed that the overexpression of PKA C $\alpha$ inhibits adipogenesis, and that the PKA inhibitor H89 $(10 \mu \mathrm{M})$ can completely reverse this inhibition, as determined by either cell morphological phenotype (Figure 5D) or the expression levels of C/EBP $\alpha$ and PPAR $\gamma$ (Figure 5E). Because cAMP and PKA activation suppresses adipogenesis, we also tested whether the inhibition of PKA activity could reverse the anti-adipogenesis effect of cAMP. In the presence of the PKA inhibitor H89 $(10 \mu \mathrm{M})$, forskolin-mediated suppression of adipogenesis was completely abolished (Figure $5 \mathrm{~F}$ and $5 \mathrm{G}$ ). Inhibition of PKA activity also abolishes 8-Br-cAMP-mediated suppression of adipogenesis (Figure $5 \mathrm{H}$ and 5I). These results further confirm that PKA is a suppressor of adipogenesis and that cAMP suppresses adipogenesis via PKA.

EGF may suppress adipogenesis through a mechanism involving the activation of $P K A$

EGF, which is essential for cell proliferation and survival, can completely suppress adipogenesis (Figure 6A). Stimulation of 3T3-L1 preadipocytes with EGF also increases PKA activity (Figure 6B). In the presence of the PKA inhibitor H89, EGF-mediated inhibition of adipogenesis was completely reversed (Figure 6C and 6D). In addition, overexpression of PKI also abolishes EGF's antiadipogenic effect (Figure 6E and 6F). The results further demonstrate a suppressive function of PKA on adipogenesis and suggest that EGF may suppress adipogenesis through a mechanism that involves the activation of PKA.

PKA and cAMP may suppress adipogenesis by modulating insulin signaling through the inactivation of IRS-1 by its serine/threonine phosphorylation

To investigate the potential mechanisms through which PKA suppresses adipogenesis, we examined the effect of the PKA activator forskolin on IRS-1 phosphorylation. Treatment with forskolin stimulates the phosphorylation of IRS-1 at ser789 in 3T3-L1 preadipocytes (Figure 7A), which is also associated with a corresponding increase in the phosphorylation of serine/threonine residues of the PKA substrate. The increased phosphorylation of IRS-1 at serine/ threonine residues was also detected by immunoprecipitation with antibody against the phospho-(serine/threonine) PKA substrate (Figure 7B), suggesting that IRS-1 is a substrate of PKA and forskolin can stimulate phosphorylation of IRS-1 by PKA at ser789. Treatment of cells with forskolin increases the formation of the complex between IRS-1 and PKA C, which further supports this possibility (Figure 7C). Further study shows that the overexpression of PKA inhibits insulin-induced tyrosine phosphorylation of IRS-1 and its activation (Figure 7D). In addition, we also determined the effect of EGF on IRS-1 phosphorylation at ser789. As shown in Figure 7E, treatment of cells with EGF increases phosphorylation of IRS-1 at ser789. Because insulin has important role in adipogenesis, the results suggest that cAMP and PKA may suppress adipogenesis by modulating insulin signaling through the inactivation of IRS-1 by its serine/threonine phosphorylation. 
A $\operatorname{Rp}-c A M P(\mu \mathrm{M}) \quad-\quad-\quad 200 \quad 500$

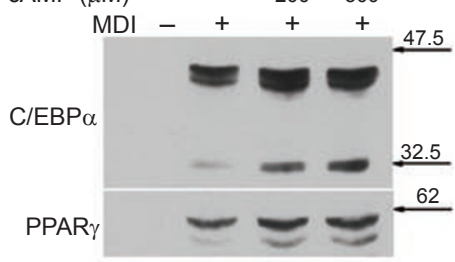

C

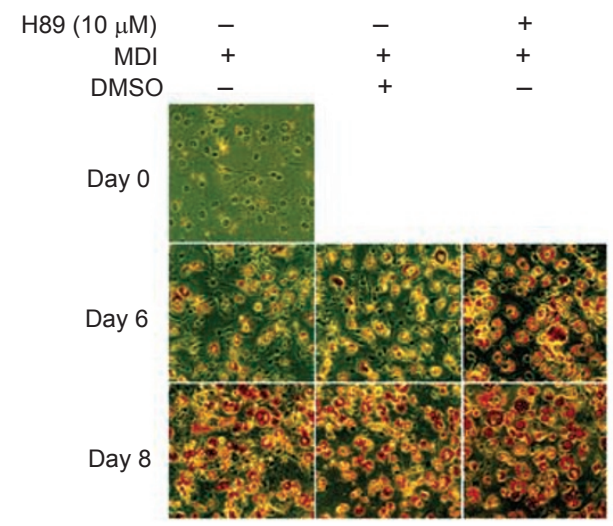

E
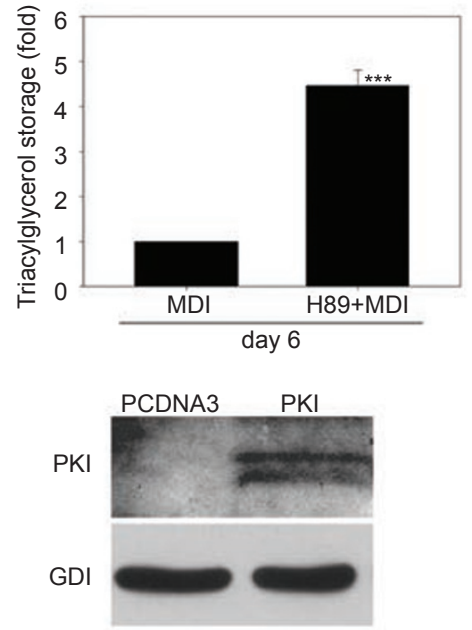

G
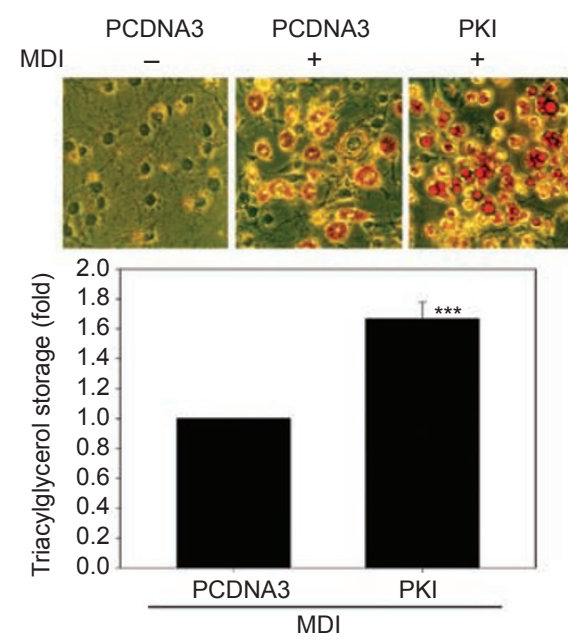

B

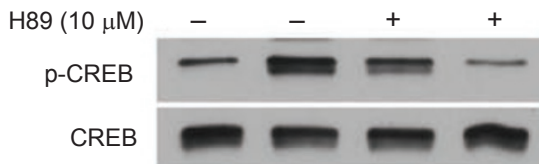

D
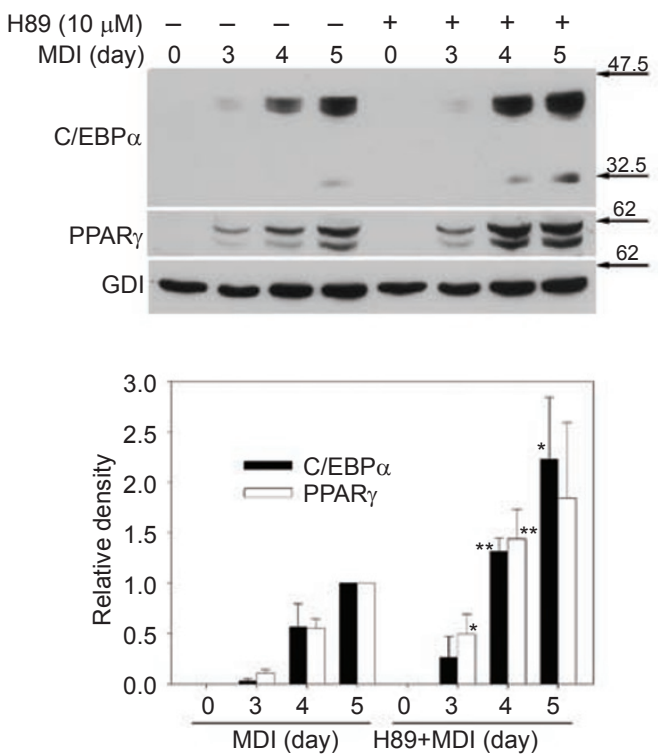

F

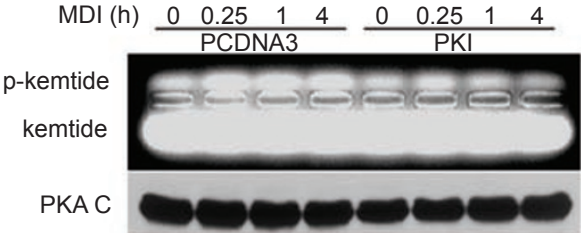

$\mathrm{H}$
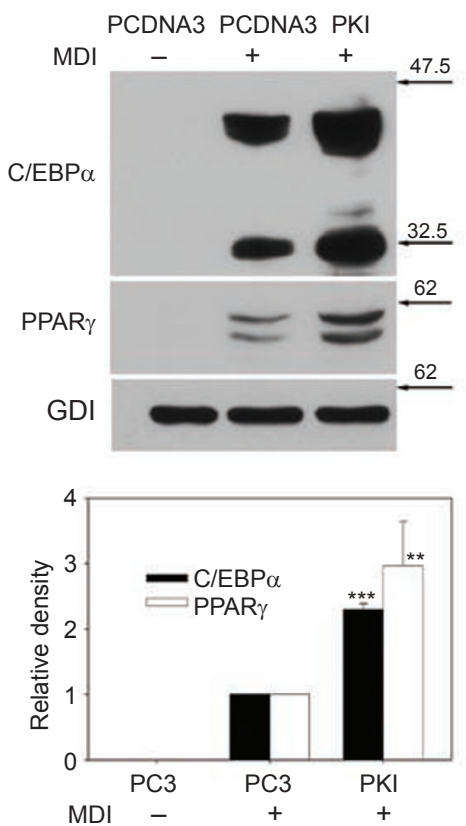


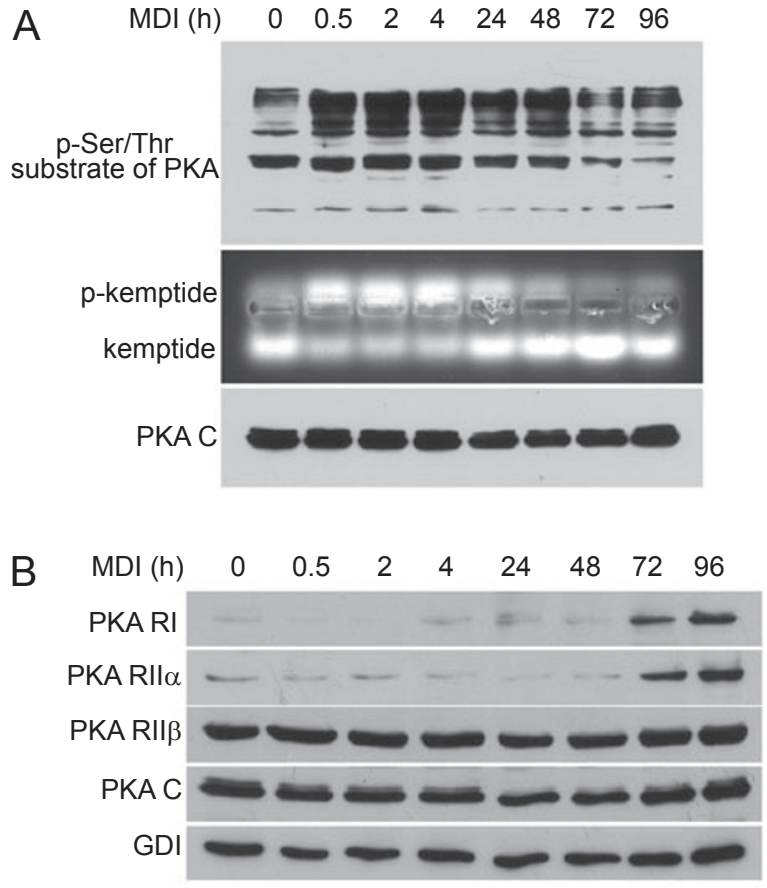

Figure 4 PKA activity and its expression levels during adipogenesis. (A) PKA activity at different time points of adipogenesis induced by MDI was determined by measuring the phosphorylation of intracellular PKA substrates (upper panel) or kemptide, a PKA substrate peptide (middle panel); the expression levels of the PKA catalytic subunit were also measured (lower panel). (B) The expression levels of the PKA regulatory subunits RI, RII $\alpha$ and RII $\beta$, as well as the PKA catalytic subunits, were determined at different time points of adipogenesis induced by MDI. The expression level of GDI was taken as a reference for sample loading control.

\section{Discussion}

The identification since the 1970 s of a series of transcriptional factors that regulate adipogenesis is a remarkable breakthrough in the study of adipocyte differentiation. For example, PPAR $\gamma$ and $\mathrm{C} / \mathrm{EBP} \alpha$ have been found to be key regulators of adipogenesis. The expression and activation of PPAR $\gamma$ and $\mathrm{C} / \mathrm{EBP} \alpha$ in preadipocytes or certain fibroblasts have crucial roles in determining the differentiation of these cells into adipocytes [22-25]. They bring about lipid accumulation and the adipocyte phenotype. It has previously been shown that cAMP and PKA are implicated in the regulation of lipid accumulation or lipolysis [26-31]. To avoid interference from the lipolysis effect of cAMP and PKA, all specific reagents used in this study (such as forskolin, SQ22536, 8-Br-cAMP and H89) were only presented in cell culture in the first 2 days of the entire induction period of adipogenesis. Under this condition, the effect of cAMP and PKA on lipolysis will not be produced.

It has been reported that several cAMP-elevating agents (such as MIX and prostaglandins/prostacyclins) can enhance adipogenesis [32-34]. However, there is a lack of direct evidence for the causative relationship between cAMP/PKA and adipogenesis. In fact, the potency of the pro-adipogenesis effect of the cAMP-elevating agents used to stimulate adipogenesis does not correlate at all with the corresponding increase in cAMP levels. Interestingly, it has also been reported that various stimulators for the cAMP pathway failed to promote the adipogenic conversion of preadipocytes [35]. In the absence of MIX, 8-Br-cAMP and forskolin notably increase DI-induced adipogenesis, whereas in the presence of MIX, cAMP analogs and forskolin suppress MDI-induced adipogenesis. These findings

Figure 3 Inhibition of PKA promotes MDI-induced adipogenesis. (A) Rp-cAMP shortens the length of induction time required for adipogenesis. 3T3-L1 preadipocytes were exposed to MDI in the absence or presence of Rp-cAMP at the indicated concentrations for $36 \mathrm{~h}$, and then incubated in fresh medium supplemented with insulin $(1 \mu \mathrm{g} / \mathrm{ml})$ for 2 days. The cells were fed with fresh medium for another 2 days. Adipogenesis was determined using the expression levels of C/EBP $\alpha$ and PPAR $\gamma$. (B) The PKA inhibitor H89 inhibits forskolin-induced PKA activity. 3T3-L1 preadipocytes were treated with or without forskolin (Forsk, $1 \mu \mathrm{M}$ ) in the presence or absence of $\mathrm{H} 89(10 \mu \mathrm{M})$ for $15 \mathrm{~min}$, cells were lysed and the phosphorylation of CREB was examined by immunoblotting blotting. (C and $\mathbf{D})$ Inhibition of PKA activity by $\mathrm{H} 89$ accelerates adipogenesis. After exposure to MDI in the absence or presence of H89 (15-min pretreatment, $10 \mu \mathrm{M})$ for 2 days, 3T3-L1 preadipocytes were incubated in fresh medium (10\% FCS) supplemented with insulin (1 $\mu \mathrm{g} / \mathrm{ml})$ for 2 days, and then were fed every second day with fresh medium (10\% FCS). Adipogenesis was determined by Oil Red O staining (B) and the expression of C/EBP $\alpha$ and PPAR $\gamma(\mathbf{C})$. For Oil Red $\mathrm{O}$ quantitative assay, cells were treated as described above and terminated on day 6 . The lower panels are the means \pm SDs from three independent experiments. ${ }^{*} P<0.05 ;{ }^{* *} P<0.01$; ${ }^{* * *} P<0.001$ versus controls (not treated with H89). ( $E$ and F) Overexpression of PKI inhibits PKA activity and stimulates adipogenesis. 3T3-L1 preadipocytes were transfected with PKI (on a PCDNA3 vector), the expression of PKI was determined by immunoblotting (E) and the effect of PKI on PKA activity was detected using the PKA substrate peptide kemptide (F). (G and H) PKI enhances adipogenesis. Adipogenesis of 3T3-L1 preadipocytes transfected with PKI or the PCDNA3 vector were induced as described in Materials and Methods. Adipogenesis was determined by Oil Red O staining $(\mathbf{G})$ or the expression levels of $\mathrm{C} / \mathrm{EBP} \alpha$ and PPAR $\gamma(\mathbf{H})$. Lower panels are the means \pm SDs from three independent experiments. ${ }^{* *} P<0.001$. 


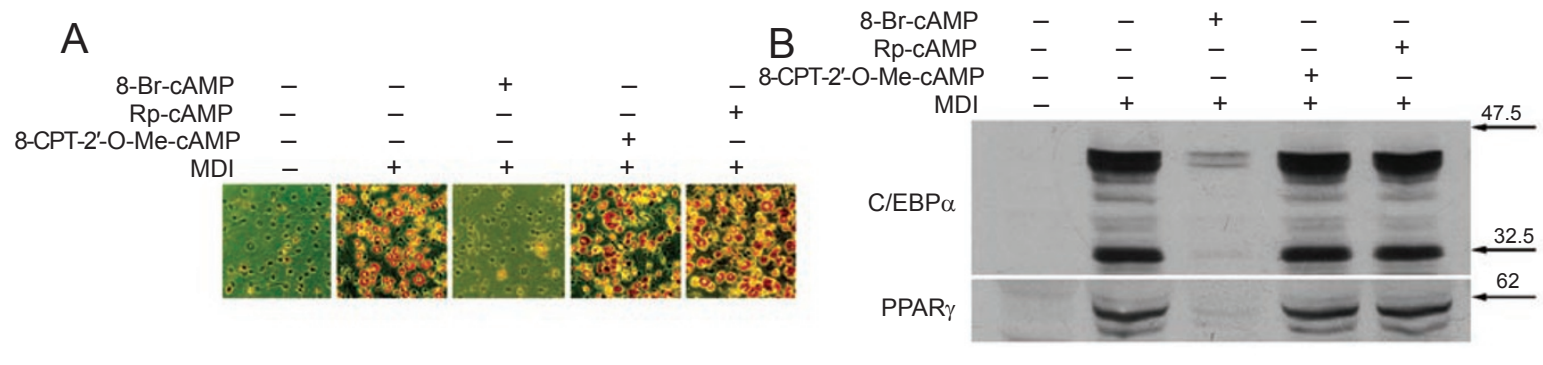

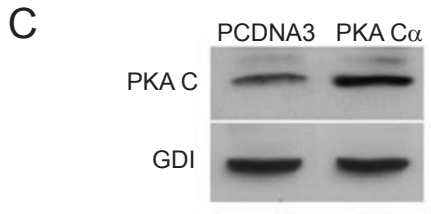

$E$

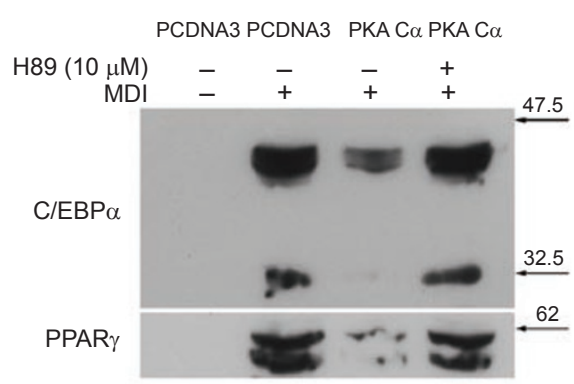

G

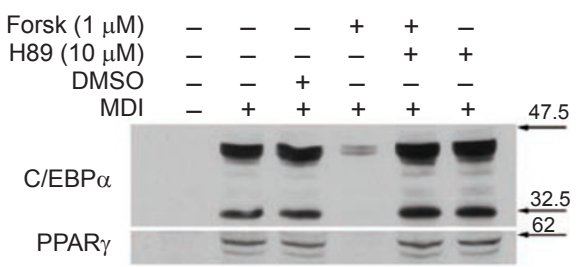

$\mathrm{D}$

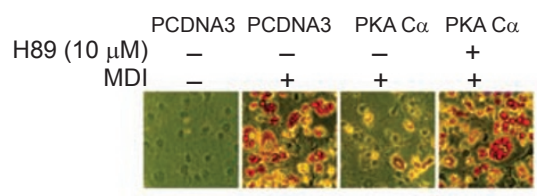

\section{$\mathrm{F}$}

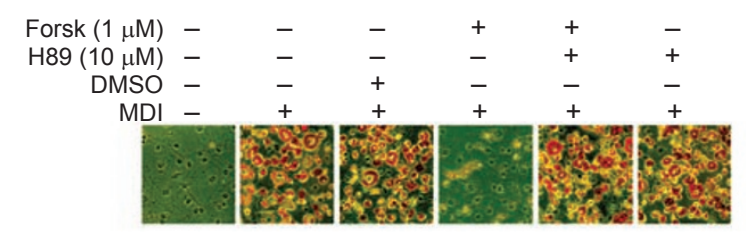

$\mathrm{H}$
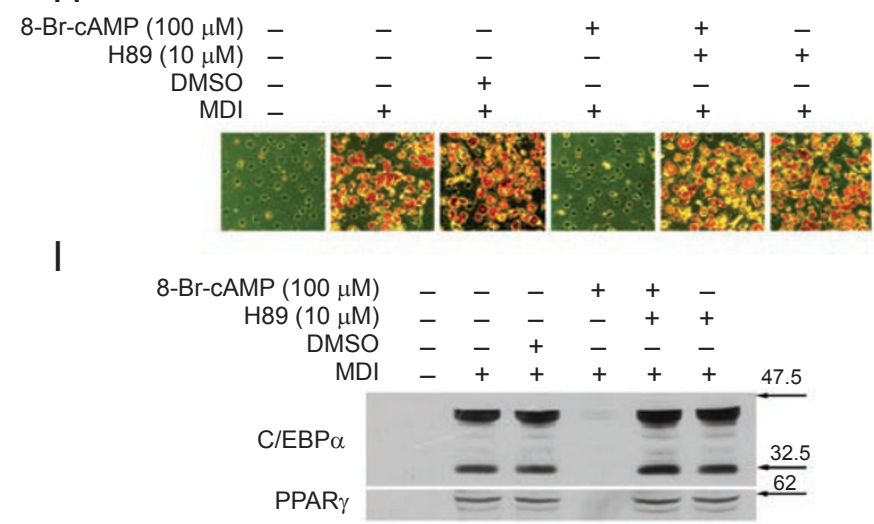

Figure 5 The effect of PKA activation or inhibition on adipogenesis. (A and B) Activation of PKA by CAMP suppresses adipogenesis. Adipogenesis of 3T3-L1 preadipocytes was induced with MDI as described in Materials and Methods in the presence or absence of 8$\operatorname{Br}$-cAMP $(100 \mu \mathrm{M})$, Rp-cAMP $(100 \mu \mathrm{M})$ or 8-CPT-2'-O-Me-cAMP $(100 \mu \mathrm{M})$. (C) Cells were transfected with the vector plasmid PCDNA3 or that containing PKA $\mathbf{C} \alpha$. The expression levels of PKA $C$ were examined by immunoblotting. (D and E) PKA inhibition abrogates the inhibitory effect of PKA C $\alpha$. Adipogenesis of cells transfected with either the plasmid vector or the wild-type PKA C $\alpha$ in the presence or absence of the PKA inhibitor H89 was determined by Oil Red O staining (D) or the expression levels of C/EBP $\alpha$ and PPAR $(\mathrm{E})$. (F-I) Cells were pretreated with or without forskolin (Forsk) or 8-Br-cAMP in the presence or absence of $\mathrm{H} 89(10 \mu \mathrm{M})$; the induction of adipogenesis was initiated with MDI as described in Materials and Methods. Adipogenesis was determined by Oil Red $\mathrm{O}$ staining $(\mathbf{F}$ and $\mathbf{H}$ ) or the expression levels of C/EBP $\alpha$ and PPAR $(\mathbf{G}$ and $\mathbf{I})$.

suggest a probable dual effect of cAMP on adipogenesis. Because forskolin-induced cAMP can be rapidly hydrolyzed by PDE in the absence of the PDE inhibitor MIX, forskolin will not cause a great increase in cAMP levels in the absence of MIX. However, in the presence of MIX, forskolin can stimulate a much stronger increase in cAMP levels. Similarly, the addition of an exogenous cAMP ana$\log$ in the presence of MIX can lead to a strong increase in cAMP levels. It is possible that a moderate increase of cAMP in the early stage of MDI induction may have a role in adipogenic induction, whereas sustained high concentrations of cAMP generate a strong inhibitory effect.

Prostaglandins/prostacyclins, which can elevate cAMP levels and have been used to stimulate adipogenesis, have been reported to activate PPAR $\gamma[36,37]$. Thus, the pro-adipogenic effect of prostaglandins/prostacyclins may be due 


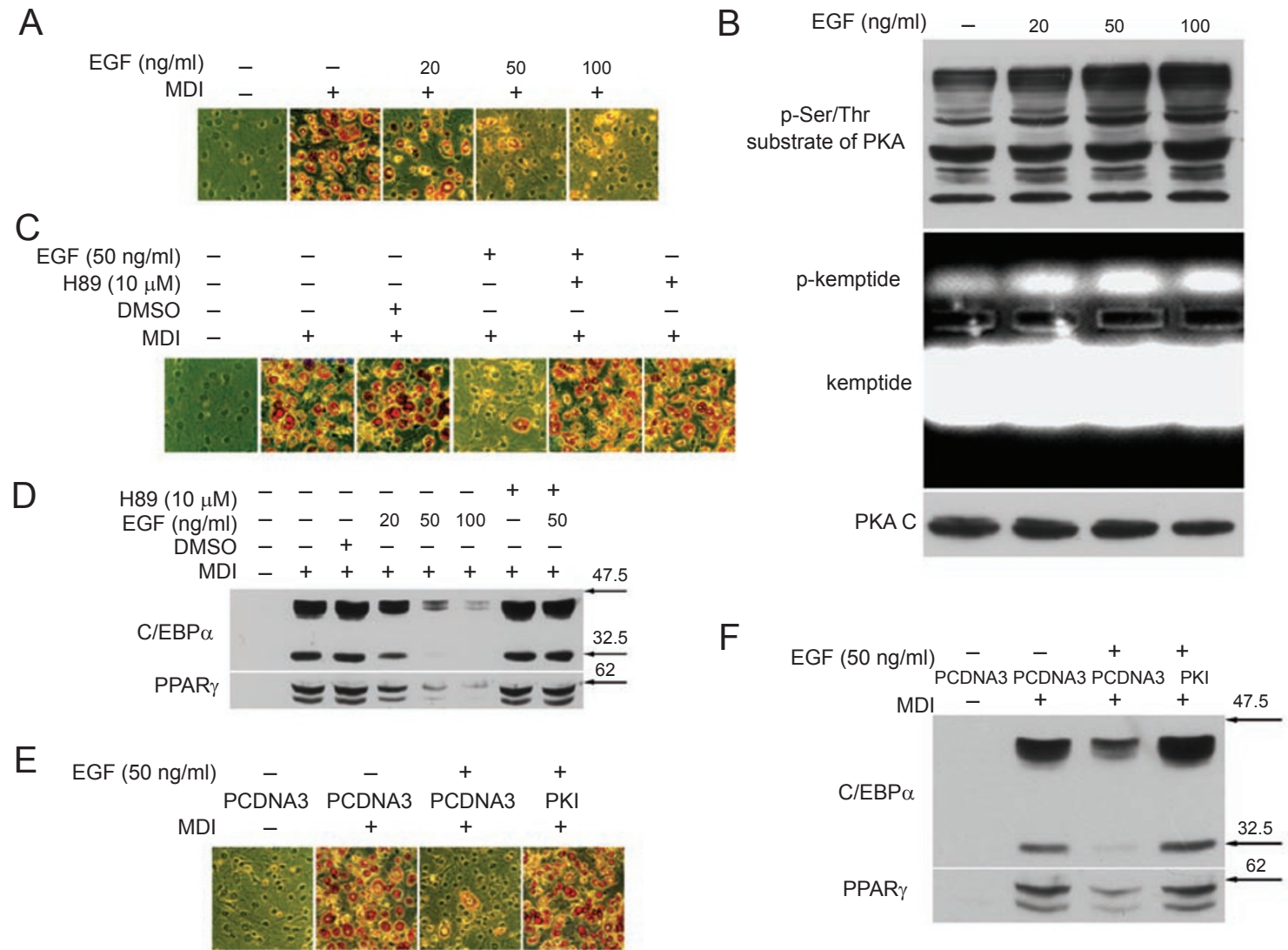

Figure 6 The effect of PKA in EGF-mediated suppression of adipogenesis. (A) EGF suppresses MDI-induced adipogenesis. MDImediated adipogenesis was conducted in the presence of EGF according to Materials and Methods and was determined by Oil Red O staining. (B) The effect of EGF on PKA activation. After cells were treated with EGF for $24 \mathrm{~h}$, PKA activity was detected using the phosphorylation levels of the intracellular PKA substrates (upper panel) or kemptide (middle panel). The expression of PKA C was also examined by immunoblotting (lower panel). (C and D) EGF-mediated suppression of adipogenesis is abolished by the PKA inhibitor H89. Cells were pretreated with or without H89 in the presence or absence of EGF for 15 min, and then were induced with MDI as described in Materials and methods. Adipogenesis was examined using Oil Red O staining (C) or the expression levels of C/EBP $\alpha$ and PPAR $\gamma$ (D). (E and F) Overexpression of PKI abolishes EGF's anti-adipogenic effect. Adipogenesis of the 3T3-L1 preadipocytes transfected with PKI or the PCDNA3 vector was induced as described in Materials and Methods. Adipogenesis was examined using Oil Red O staining (E) or the expression levels of C/EBP $\alpha$ and PPAR $\gamma(F)$.

to their activation of PPAR $\gamma$. As an inducer of adipogenesis, MIX stimulates an increase in intracellular cAMP levels, but this does lead to the conclusion that PKA functions as a stimulator of adipogenesis. As a non-specific inhibitor of phosphodiesterases, the signaling induced by MIX is closely related to that induced by phosphodiesterases. At present, more than 50 variants of the mammalian phosphodiesterase isoenzyme, which shows differential hydrolytic activities on cAMP and cGMP, have been identified [38]. This suggests another possibility that the cellular responses to MIX could be different owing to the different cell types or to the different expression patterns of phosphodiesterases. It has been reported that 3T3-L1 preadipocytes express phosphodiesterase 3B (PDE3B) [39, 40], a phosphodiesterase that hydrolyzes both cAMP and cGMP, which indicates that MIX increases not only cAMP but also cGMP during adipogenesis. TNF- $\alpha$ can elevate cAMP level but are unable to stimulate adipogenesis [29]. TNF- $\alpha$ has been shown to be involved in the inhibition of adipogenesis [41]. Although the precise mechanism that underlies the effect of MIX on adipogenesis was not investigated in the present study, our data demonstrate that cAMP at high concentrations functions as a potent suppressor for adipogenesis that is induced by MDI, which is of interest in the study of the signaling mechanisms of adipogenesis.

The finding that MDI-induced adipogenesis is inhibited by 8-bromo-cAMP, forskolin and constitutive ectopic PKA C $\alpha$ expression demonstrates that PKA activation or increased PKA expression suppresses adipogenesis. The fact that overexpression of PKI or Rp-cAMP reduces the 


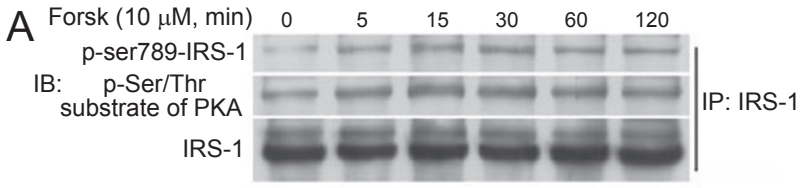

B

$\begin{array}{cccccc}\text { Forsk }(10 \mu \mathrm{M}, \min ) & 0 & 0 & 15 & 60 & 120 \\ \text { IB: IRS-1 } & & & & & \\ \text { anti-p-Ser/Thr } & & & & & \\ \text { IP: substrate of PKA } & - & + & + & + & + \\ \text { IgG (control) } & + & - & - & - & -\end{array}$

C
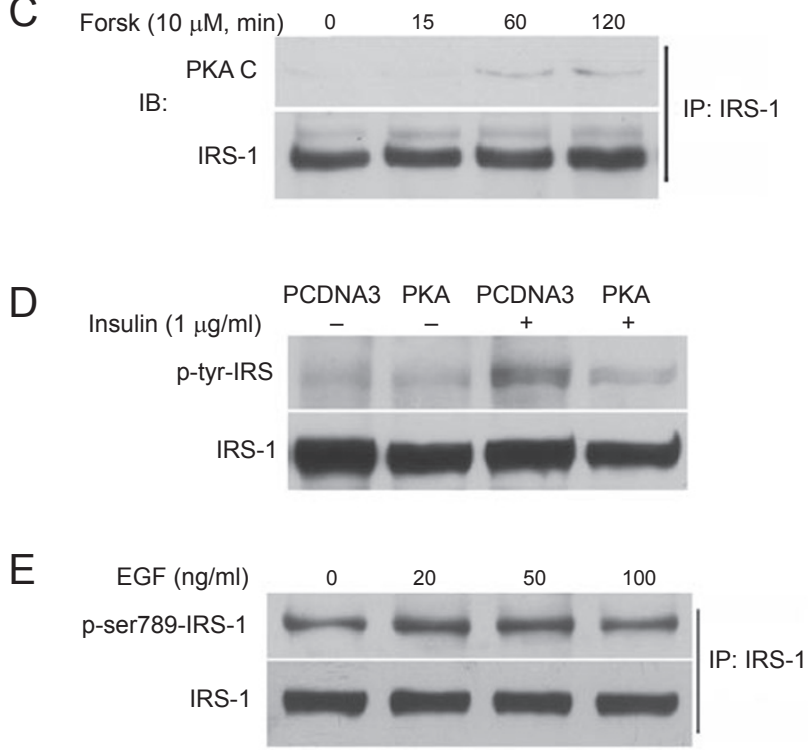

Figure 7 Regulation of IRS-1 phosphorylation at ser789. (A) Cell lysates are immunoprecipitated by an IRS-1-specific antibody. Forskolin-induced IRS-1 phosphorylation was detected using either a specific antibody against phosphorylated IRS-1 at the ser789 residue or a serine/threonine-phosphorylated PKA substrate. (B) Cell lysates are immunoprecipitated by a specific antibody against the serine/threonine-phosphorylated PKA substrate. Increased phosphorylated IRS-1 protein levels were detected by an IRS-1specific antibody. (C) Forskolin induces the association between IRS-1 and PKA C. (D) Overexpression of PKA inhibits insulin-induced tyrosine phosphorylation of IRS-1. 3T3-L1 cells transfected with a vector (PCDNA3) or PKA were stimulated with or without insulin $(1 \mu \mathrm{g} / \mathrm{ml})$ for $30 \mathrm{~min}$ and subjected to immunoblotting tyrosine phosphorylation of IRS-1. (E) EGF stimulates phosphorylation of IRS-1 at ser789. After being treated with EGF for 24 h, 3T3-L1 preadipocytes were lysed and subjected to immunoprecipitation to determine IRS-1 phosphorylation at ser789.

general MDI induction time (from 48 to $36 \mathrm{~h}$ ) and that $\mathrm{H} 89$ reduces the time span required for full adipogenesis procession (from 8 to 6 days) indicates that inhibition of PKA accelerates adipogenesis. Overexpression of PKI or
H89 can abolish the anti-adipogenic effect produced by EGF, 8-bromo-cAMP, forskolin and expression of constitutive ectopic PKA $\mathrm{C} \alpha$, which strongly suggests that PKA is a key regulator in the control of adipogenesis. All these results demonstrate that PKA acts as a suppressor for adipogenesis and cAMP suppresses adipogenesis through PKA. The observation that PKA activity was significantly stimulated during the first 2 days of adipogenesis may appear to be perplexing. This is possibly a side effect of MIX in the early stage of induction. MIX could be a much more powerful inducer if it did not activate PKA because inhibition of MDI-induced PKA activity by overexpression of PKI and treatment of cells with Rp-cAMP or H89 accelerates adipogenesis. On the other hand, PKA activity was only increased during the early induction period of adipogenesis, which started to fall $24 \mathrm{~h}$ after the MDI treatment and dropped to the base level after $72 \mathrm{~h}$. During the normal process of adipogenesis induced by MDI, other signaling events must have been activated or inactivated that canceled out or reduced the anti-adipogenic effect generated by PKA activation.

Because the decreased PKA activity on the third day of induction of adipogenesis is correlated with increased expression of the RI and RII $\alpha$ subunits of PKA (Figure 4), this further suggests that PKA has a suppressive role in adipogenesis. Inhibition of PKA may become important 2-3 days after induction, because the real adipogenesis takes place after that period. The RII $\beta$ subunit is highly expressed in both preadipocytes and adipocytes. Interestingly, only expression of the RI and RII $\alpha$ subunits of PKA is increased, with no change in the expression levels of the RII $\beta$ subunit. Although the mechanism is not clear, it appears that the RI and RII $\alpha$ subunits of PKA are more important than other subunits of PKA in the control of adipogenesis. The inhibitory function of the RII $\beta$ subunit may be restricted or modified by certain factors, such as inhibitory anchoring proteins and intracellular compartmentalization.

The exact role of EGF in adipogenesis has not been well described. It has been reported that EGF has a dual effect on fat synthesis $[42,43]$. It decreases adipogenesis in undifferentiated preadipocytes but increases adipogenesis in differentiated adipocytes [44]. Pref-1, a member of the EGF-like family proteins, has been implicated in the suppression of adipogenesis. Pref-1 is highly expressed in 3T3-L1 preadipocytes, but is undetectable in mature fat cells, and this downregulation has been shown to be required for adipocyte differentiation $[45,46]$. Interestingly, we found that EGF also inhibits the differentiation of 3T3-L1 preadipocytes. EGF-mediated suppression of adipogenesis is associated with PKA activation. It is striking that this suppressive effect of EGF can be abolished by inhibiting PKA with $\mathrm{H} 89$ or overexpression of PKI, 
indicating that PKA also has a crucial role in the EGFmediated suppression of adipogenesis. Because Pref-1 has been characterized as an inhibitor of adipogenesis, the fact that EGF suppresses adipogenesis through PKA also suggests that EGF-like family proteins, such as Pref-1, may exert their effects on adipogenesis through the activation of PKA. Furthermore, it has been reported that H89 can block the anti-adipogenesis effect of arachidonic acid [47]. All these data indicate that PKA has a crucial role in suppressing adipocyte differentiation. In this report, we found that IRS-1 acts as a substrate of PKA, and that cAMP and PKA can inhibit insulin signaling through the phosphorylation of IRS-1 at ser789 and the inactivation of IRS- 1 . Since IRS-1 has an essential role during adipogenesis, the results suggest a modulatory function of cAMP and PKA on the insulin-mediated adipogenic effect through IRS-1. These data suggest that cAMP and PKA can be taken as novel targets for developing new reagents in treating obesity and diabetes.

In conclusion, our study demonstrates that cAMP analogs, cAMP-elevating agent forskolin and PKA activation can inhibit MDI-induced adipogenesis. Inhibition of PKA promotes adipogenesis by reducing the time required for adipogenic induction. Forskolin or 8-Br-cAMP-mediated inhibition of adipogenesis can be abolished by inhibiting PKA activity, indicating that PKA has an important role in the suppression of adipogenesis. In addition, the data also show that PKA-mediated suppression of preadipocyte differentiation is associated with the inactivation of IRS-1.

\section{Materials and Methods}

\section{Materials}

The cell culture reagents newborn calf serum (NCS) and fetal bovine serum (FCS) were purchased from Gibco BRL of Invitrogen Corporation (Carlsbad, CA, USA). MDI, Oil Red O and EGF were purchased from Sigma (St Louis, MO, USA). H89, G418, forskolin, 8-Br-cAMP and Rp-cAMP were purchased from Calbiochem (La Jolla, CA, USA). 8-CPT-2'-O-Me-cAMP was purchased from ALEXIS Biochemicals (San Diego, CA, USA). SQ22536 was purchased from Biomol (Plymouth Meeting, PA, USA). Rabbit anti-PPAR $\gamma, \mathrm{C} / \mathrm{EBP} \alpha$, IRS-1, and goat anti-phospho-tyr612-IRS, horseradish peroxidase (HRP)-conjugated anti-mouse, anti-rabbit and anti-goat antibodies were purchased from Santa Cruz Biotechnology (Delaware, CA, USA). Antibodies against PKA C, PKA RI, PKA RII and PKA RII from mouse were purchased from BD Biosciences (San Jose, CA, USA). Rabbit anti-guanine nucleotide dissociation inhibitor (GDI) antibody was purchased from Zymed of Invitrogen Corporation (Carlsbad, CA, USA). Rabbit antibodies against phospho-(serine/threonine) PKA substrate and phospho-IRS-1 (ser789) were from Cell Signaling (Beverly, MA, USA).

\section{Cell culture}

3T3-L1 preadipocytes were cultured in Dulbecco's modified Eagle's medium (DMEM, Gibco BRL) supplemented with 10\% (v/v)
NCS, $100 \mathrm{U} / \mathrm{ml}$ penicillin, and $100 \mathrm{mg} / \mathrm{ml}$ streptomycin (Gibco BRL), at $37^{\circ} \mathrm{C}$ in a humidified atmosphere of $90 \%$ air and $10 \% \mathrm{CO}_{2}$. Medium was renewed every 2-3 days until confluence was reached.

\section{Adipogenesis procedure}

After 2 days' confluence, the cells were incubated in fresh medium containing $10 \%$ FCS. Cells were then treated with MIX $(0.5 \mathrm{mM})$, dexamethasone (DEX, $1 \mu \mathrm{M})$ and insulin $(1 \mu \mathrm{g} / \mathrm{ml})$ to initiate the differentiation (designated as day 0 ). After exposure to MDI for 2 days, cells were incubated in fresh medium (10\% FCS) containing only insulin $(1 \mu \mathrm{g} / \mathrm{ml})$ for a further 2 days. The cells were then fed every second day with fresh medium $(10 \%$ FCS). Triglyceride droplets were visible on day 4 , and the cells were fully differentiated into adipocytes on day 8 (over $90 \%$ of the plate). H89, forskolin, 8-Br-cAMP, Rp-cAMPS, 8-CPT-2'-O-Me-cAMP, SQ22536 and EGF were added 15 min prior to the MDI induction. The cells were incubated in the presence of MDI and indicated agents typically for a 48-h period only, unless otherwise indicated.

\section{Cell transfection}

PKA C $\alpha$ and PKI in PCDNA3 were constructed as follows. PKA C $\alpha$ and PKI cDNAs that ended with the relevant restricted enzyme sites were cloned from a mouse testicle cDNA library with the relevant primer pairs: 5'-GCaagcttATGGGCAACGCCGCCG-3' and 5'-GCggatccCTAAAACTCAGTAAACTCCTTG-3' for PKA $\mathrm{C} \alpha$; and 5'-GGCaagcttATGACTGATGTGGAAACTACG-3' and 5'-GGCtctagaTTAGCTTTCAGACTTGGCTGCTTC-3' for PKI. After confirmation using DNA sequencing, the cloned cDNAs were ligated into the PCDNA3 vector. The cells were transfected with the Lipofectamine ${ }^{\mathrm{TM}}$ Reagent according to the manufacturer's instructions. Cells grown to a suitable density for $24 \mathrm{~h}$ after seeding are usually necessary for high efficiency transfection. In detail, 3T3-L1 fibroblasts were grown to $80 \%$ confluence on $30-\mathrm{mm}$ plates and washed with DMEM before adding the transfection reagents. Two micrograms of plasmid DNA (in $100 \mu 1$ DMEM) and $6 \mu \mathrm{Li}$ pofectamine ${ }^{\mathrm{TM}}$ Reagent (in $100 \mu \mathrm{l}$ DMEM) were mixed gently and incubated at room temperature for $30 \mathrm{~min}$, followed by dilution with DMEM to $1 \mathrm{ml}$ before beginning transfection. Twelve hours after transfection, cells were incubated in fresh culture medium for $72 \mathrm{~h}$, then trypsinized and reseeded in fresh culture medium supplemented with $600 \mu \mathrm{g} / \mathrm{ml} \mathrm{G} 418$. Medium containing G418 was changed every 2-4 days, and cells were cultured for 2-3 weeks to selectively remove the non-transfected cells. When necessary, cells were reseeded onto new plates to ensure even distribution.

\section{Immunoprecipitation}

Cells on 60-mm plates were lysed in $0.4 \mathrm{ml}$ of ice-cold lysis buffer (50 mM HEPES pH 7.4, $5 \mathrm{mM}$ EDTA, $50 \mathrm{mM} \mathrm{NaCl}, 1 \%$ Triton $\mathrm{X}-100,50 \mathrm{mM} \mathrm{NaF}, 1 \mathrm{mM} \mathrm{Na} \mathrm{VO}_{4}, 10 \mathrm{mM} \mathrm{Na}_{4} \mathrm{P}_{2} \mathrm{O}_{7} \cdot \mathrm{H}_{2} \mathrm{O}, 5 \mathrm{mg} / \mathrm{ml}$ aprotinin, $5 \mathrm{mg} / \mathrm{ml}$ leupeptin and $1 \mathrm{mM} \mathrm{PMSF}$ ). The lysates were centrifuged at $12000 \times g$ for $15 \mathrm{~min}$. The supernatants were collected and subjected to protein quantification with a Bio-Rad protein determination kit. Supernatants with equal protein were treated by the following steps. They were pre-cleared with $30 \mu \mathrm{l} 50 \%$ beads for $2 \mathrm{~h}, 4 \mu \mathrm{l}$ indicated antibody (IRS-1, p-serine/threonine substrate of PKA or rabbit IgG; about $1 \mu \mathrm{g}$ ) was added and rotated for $2 \mathrm{~h}$, $30 \mu 150 \%$ beads were added and then they were rotated overnight. The precipitates were washed three times with lysis buffer and then $35 \mu \mathrm{l}$ of $1 \times$ loading buffer was added. Samples were subjected to 
SDS-polyacrylamide gel electrophoresis (SDS-PAGE) and immunoblotting.

\section{Immunoblotting}

Cells were lysed in $0.4 \mathrm{ml}$ of ice-cold lysis buffer. The lysates were centrifuged at $12000 \times g$ for $15 \mathrm{~min}$. The supernatants were collected and subjected to protein quantification with a Bio-Rad protein determination kit. Equal amounts of protein (about $40 \mu \mathrm{g}$ ) and supernatants were separated by SDS-PAGE and transferred to nitrocellulose membranes (Hybond ECL, Amersham Life Sciences). The membranes were blocked with $5 \%$ non-fat dry milk in tris-buffered saline containing $0.1 \%$ tween-20 (TBS-T) and subsequently incubated with antibodies (1:2 000) against PPAR $\gamma, \mathrm{C} / \mathrm{EBP} \alpha, \mathrm{PKA}$ C, PKA RI, PKA RII $\alpha$, PKA RII $\beta$, Phospho-(serine/threonine) PKA substrate and GDI. Specific protein bands were detected using HRP-conjugated goat anti-mouse or anti-rabbit $\operatorname{IgG}(1: 4$ 000) and visualized using ECL reagents (Pierce). The protein expression levels of PPAR $\gamma$ and $\mathrm{C} / \mathrm{EBP} \alpha$ were analyzed on day 6 of differentiation, unless otherwise indicated. In some cases, the results were quantified by calculating the optical density of the relevant bands detected. To get the relative values of the western blot bands, the density of the fully differentiated cells from the sample by the normal differentiation procession (the control differentiated cells) was set as 1 . The nitrocellulose membrane was stripped with a strip buffer $(62.5 \mathrm{mM}$ Tris- $\mathrm{HCl}, \mathrm{pH} 6.7,100 \mathrm{mM} 2$-mercaptoethanol and $2 \% \mathrm{SDS}$ ) at $65^{\circ} \mathrm{C}$ for $45 \mathrm{~min}$ and probed with other antibodies if necessary.

\section{cAMP measurement}

After carrying out the indicated treatment, 3T3-L1 preadipocytes on 60 -mm plates were lysed with $0.4 \mathrm{ml} 0.1 \mathrm{M} \mathrm{HCl}$ and the intracellular level of cAMP was detected with a Cyclic AMP Enzyme Immunoassay kit (AK-205, Biomol) according to the vendor's instructions.

\section{PKA activity assay}

A PKA kinase assay was carried out with a non-radioactive PKA assay kit (Promega). Phosphorylation by PKA of its specific peptide substrate alters the peptide's net charge from +1 to -1 , which allows the phosphorylated and non-phosphorylated forms of the substrate to be rapidly separated on agarose gel. The phosphorylated substrates migrate towards the positive electrode whereas the non-phosphorylated substrates migrate towards the negative electrode. Phosphorylation of the PKA substrate was detected by immunoblotting with a phospho-(serine/threonine) PKA substrate antibody.

\section{Oil Red O staining}

Fully differentiated 3T3-L1 cells (day 8) were stained with Oil Red $\mathrm{O}$ according to the method outlined here. Cells were washed three times with PBS, then fixed with $3.7 \%$ formaldehyde in PBS for $2 \mathrm{~min}$, washed three times with $\mathrm{H}_{2} \mathrm{O}$ and then stained with Oil Red O solution ( $0.3 \%$ Oil Red O in 100\% isopropanol, filtered) for $1 \mathrm{~h}$. Cells were then washed three times with and maintained in $\mathrm{H}_{2} \mathrm{O}$. The phenotypic change of adipogenesis was observed under a microscope. For a quantitative assay, Oil Red O dye was extracted with isopropanol and the absorbance was measured at $510 \mathrm{~nm}$ with a spectrophotometer.

\section{Statistical analysis}

Results were presented as the means \pm standard deviations (SD) for the number of experiments indicated. For statistical analysis, Student's $t$-test was used. Differences were considered significant at a level of $P<0.05$.

\section{Acknowledgments}

We thank our colleagues Kan Liao for the 3T3-L1 preadipocyte cell lines and Xinchao Pan for technical assistance in preparing this manuscript. We also thank other members of the laboratory for many helpful discussions. This work was supported by the Natural Science Foundation of China (30623003 and 30421005), the Shanghai Research and Development Base (06DZ22032) and the Science and Technology Committee of Shanghai Municipality (058014578).

\section{References}

1 Bennett CN, Ross SE, Longo KA, et al. Regulation of Wnt signaling during adipogenesis. J Biol Chem 2002; 277:30998-31004.

2 Kopelman PG. Obesity as a medical problem. Nature 2000; 404:635-643.

3 Green H, Meuth M. An established pre-adipose cell line and its differentiation in culture. Cell 1974; 3:127-133.

4 MacDougald OA, Lane MD. Transcriptional regulation of gene expression during adipocyte differentiation. Annu Rev Biochem 1995; 64:345-373.

5 Lowell BB. PPAR $\gamma$ : an essential regulator of adipogenesis and modulator of fat cell function. Cell 1999; 99:239-242.

6 Rosen ED, Spiegelman BM. Molecular regulation of adipogenesis. Annu Rev Cell Dev Biol 2000; 16:145-171.

7 de Rooij J, Zwartkruis FJ, Verheijen MH, et al. Epac is a Rap1 guanine-nucleotide-exchange factor directly activated by cyclic AMP. Nature 1998; 396:474-477.

8 DiPilato LM, Cheng X, Zhang J. Fluorescent indicators of cAMP and Epac activation reveal differential dynamics of cAMP signaling within discrete subcellular compartments. Proc Natl Acad Sci USA 2004; 101:16513-16518.

9 Osterrieder W, Brum G, Hescheler J, Trautwein W, Flockerzi $\mathrm{V}$, Hofmann F. Injection of subunits of cyclic AMP-dependent protein kinase into cardiac myocytes modulates $\mathrm{Ca}^{2+}$ current. Nature 1982; 298:576-588.

10 Costa MR, Casnellie JE, Catterall WA. Selective phosphorylation of the alpha subunit of the sodium channel by cAMP-dependent protein kinase. J Biol Chem 1982; 257:7918-7921.

11 Shabb JB. Physiological substrates of cAMP-dependent protein kinase. Chem Rev 2001; 101:2381-2411.

12 Smith PJ, Wise LS, Berkowitz R, Wan C, Rubin CS. Insulin-like growth factor-I is an essential regulator of the differentiation of 3T3-L1 adipocytes. J Biol Chem 1988; 263:9402-9408.

13 Sun XJ, Rothenberg P, Kahn CR, et al. Structure of the insulin receptor substrate IRS-1 defines a unique signal transduction protein. Nature 1991; 352:73-77.

14 Miki H, Yamauchi T, Suzuki R, et al. Essential role of insulin receptor substrate 1 (IRS-1) and IRS-2 in adipocyte differentiation. Mol Cell Biol 2001; 21:2521-2532.

15 Horike N, Takemori H, Katoh Y, et al. Adipose-specific expres- 
sion, phosphorylation of ser794 in insulin receptor substrate-1, and activation in diabetic animals of salt-inducible kinase-2. $J$ Biol Chem 2003; 278:18440-18447.

16 Katoh Y, Takemori H, Horike N, et al. Salt-inducible kinase (SIK) isoforms: their involvement in steroidogenesis and adipogenesis. Mol Cell Endocrinol 2004; 217:109-112.

17 Liu YF, Herschkovitz A, Boura-Halfon S, et al. Serine phosphorylation proximal to its phosphotyrosine binding domain inhibits insulin receptor substrate 1 function and promotes insulin resistance. Mol Cell Biol 2004; 24:9668-9681.

18 Araujo EP, De Souza CT, Gasparetti AL, et al. Short-term in vivo inhibition of insulin receptor substrate-1 expression leads to insulin resistance, hyperinsulinemia, and increased adiposity. Endocrinology 2005; 146:1428-1437.

19 Evert M, Sun J, Pichler S, Slavova N, Schneider-Stock R, Dombrowski F. Insulin receptor, insulin receptor substrate-1, Raf-1, and Mek-1 during hormonal hepatocarcinogenesis by intrahepatic pancreatic islet transplantation in diabetic rats. Cancer Res 2005; 64:8093-8100.

20 Temple KA, Basko X, Allison MB, Brady MJ. Uncoupling of 3T3-L1 gene expression from lipid accumulation during adipogenesis. FEBS Lett 2007; 581:469-474.

21 Roncari DA, Van RL. Promotion of human adipocyte precursor replication by 17 beta-estradiol in culture. J Clin Invest 1978; 62:503-508.

22 Shao D, Lazar MA. Peroxisome proliferator activated receptor $\gamma$, CCAAT/enhancer-binding protein $\alpha$, and cell cycle status regulate the commitment to adipocyte differentiation. $J$ Biol Chem 1997; 272:21473-21478.

23 Zhang J, Fu M, Cui T, et al. Selective disruption of PPAR $\gamma 2$ impairs the development of adipose tissue and insulin sensitivity. Proc Natl Acad Sci USA 2004; 101:10703-10708.

24 Zhang JW, Tang QQ, Vinson C, Lane MD. Dominant-negative $\mathrm{C} / \mathrm{EBP}$ disrupts mitotic clonal expansion and differentiation of 3T3-L1 preadipocytes. Proc Natl Acad Sci USA 2004; 101:4347.

25 Kim SW, Her SJ, Kim SY, Shin CS. Ectopic overexpression of adipogenic transcription factors induces transdifferentiation of MC3T3-E1 osteoblasts. Biochem Biophys Res Commun 2005; 327:811-819.

26 Brown JD, Stone DB. The mechanism of action antilipolytic agents: a comparison of the effects of PGE1, insulin, tolbutamide, and phenformin on lipolysis induced by dibutryl cyclic AMP. Diabetes 1968; 17:304-305.

27 Spiegelman BM, Green H. Cyclic AMP-mediated control of lipogenic enzyme synthesis during adipose differentiation of 3 T3 cells. Cell 1981; 24:503-510.

28 Planas JV, Cummings DE, Idzerda RL, McKnight GS. Mutation of the RII $\beta$ subunit of protein kinase A differentially affects lipolysis but not gene induction in white adipose tissue. $J$ Biol Chem 1999; 274:36281-36287.

29 Zhang HH, Halbleib M, Ahmad F, Manganiello VC, Greenberg AS. Tumor necrosis factor- $\alpha$ stimulates lipolysis in differentiated human adipocytes through activation of extracellular signal-related kinase and elevation of intracellular cAMP. Diabetes 2002; 51:2929-2935.

30 Zhang HH, Souza SC, Muliro KV, Kraemer FB, Obin MS, Greenberg AS. Lipase-selective functional domains of perilipin
A differentially regulate constitutive and protein kinase A-stimulated lipolysis. J Biol Chem 2003; 278:51535-51542.

31 Yin W, Mu J, Birnbaum MJ. Role of AMP-activated protein kinase in cyclic AMP-dependent lipolysis in 3T3-L1 adipocytes. $J$ Biol Chem 2003; 278:43074-43080.

32 Williams IH, Polakis SE. Differentiation of 3T3-L1 fibroblasts to adipocytes. The effect of indomethacin, prostaglandin E1 and cyclic AMP on the process of differentiation. Biochem Biophys Res Commun 1977; 77:175-186.

33 Student AK, Hsu RY, Lane MD. Induction of fatty acid synthetase synthesis in differentiating 3T3-L1 preadipocytes. J Biol Chem 1980; 255:4745-4750.

34 Hopkins NK, Gorman RR. Regulation of 3T3-L1 fibroblast differentiation by prostacyclin (prostaglandin I2). Biochim Biophys Acta 1981; 663:457-466.

35 Boone C, Grégoire F, Remacle C. Various stimulators of the cyclic AMP pathway fail to promote adipose conversion of porcine preadipocytes in primary culture. Differentiation 1999; 64:255-262.

36 Forman BM, Tontonoz P, Chen J, Brun RP, Spiegelman BM, Evans RM. 15-Deoxy-d12, 14-prostaglandin J2 is a ligand for the adipocyte determination factor PPAR $\gamma$. Cell 1995; 83:803-812.

37 Kliewer SA, Lenhard JM, Willson TM, Patel I, Morris DC, Lehmann JM. A prostaglandin J2 metabolite binds peroxisome proliferator-activated receptor $\gamma$ and promotes adipocyte differentiation. Cell 1995; 83:813-819.

38 Maurice DH, Palmer D, Tilley DG, et al. Cyclic nucleotide phosphodiesterase activity, expression, and targeting in cells of the cardiovascular system. Mol Pharmacol 2003; 64:533-546.

39 Kitamura T, Kitamura Y, Kuroda S, et al. Insulin-induced phosphorylation and activation of cyclic nucleotide phosphodiesterase 3B by the serine-threonine kinase Akt. Mol Cell Biol 1999; 19:6286-6296.

40 Mei J, Holst LS, Landström TR, et al. C(2)-ceramide influences the expression and insulin-mediated regulation of cyclic nucleotide phosphodiesterase 3B and lipolysis in 3T3-L1 adipocytes. Diabetes 2002; 51:631-637.

41 Torti FM, Torti SV, Larrick JW, Ringold GM. Modulation of adipocyte differentiation by tumor necrosis factor and transforming growth factor $\beta$. J Cell Biol 1989; 108: 1105-1113.

42 Adachi H, Kurachi H, Homma H, et al. Epidermal growth factor promotes adipogenesis of 3T3-L1 cell in vitro. Endocrinology 1994; 135:1824-1830.

43 Serrero G, Mills D. Physiological role of epidermal growth factor on adipose tissue development in vivo. Proc Natl Acad Sci USA 1991; 88: 3912-3916.

44 Adachi H, Kurachi H, Homma H, et al. Epidermal growth factor promotes adipogenesis of 3T3-Ll cell in vitro. Endocrinology 1994; 135:1824-1830.

45 Smas CM, Sul HS. Pref-1, a protein containing EGF-like repeats, inhibits adipocyte differentiation. Cell 1993; 73:725-734.

46 Zhang $\mathrm{H}$, Noohr J, Jensen $\mathrm{CH}$, et al. Insulin-like growth factor1/insulin bypasses Pref-1/FA1-mediated inhibition of adipocyte differentiation. J Biol Chem 2003; 278:20906-20914.

47 Petersen RK, Jorgensen C, Rustan AC, et al. Arachidonic aciddependent inhibition of adipocyte differentiation requires PKA activity and is associated with sustained expression of cyclooxygenases. J Lipid Res 2003; 44:2320-2330. 